\title{
Síndrome hemofagocítico secundario: reporte de 5 casos
}

\author{
CAMILA PEÑA ${ }^{1}$, XIMENA VALLADARES $^{2}$,

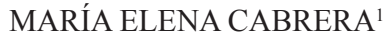

\section{Secondary hemophagocytic syndrome: report of 5 cases}

Secondary hemophagocytic syndrome (HFS) is an uncommon entity with a high mortality rate in adults, if no therapy is given. It is characterized by a severe hipercytokinemia due to a highly stimulated but ineffective immune system. The principal causes are infections, malignancy or autoimmune diseases. It appears as a serious illness, similar to a multiorgan failure. Treatment is not well defined. We report five patients with HFS, aged 17 to 51 years (three females). The etiology was onco-hematological in three patients. In two patients, the diagnosis was performed during necropsy. One case was due to cytomegalovirus (CMV) infection in a hepatic transplant patient and the other, due to parenteral lipid administration. All presented fever, cytopenia, hepatosplenomegaly and hemophagocytosis. Four of them required admission in an Intensive Care Unit. All received different treatment modalities. Only one survived. Median survival time was 75 days. In conclusion, HFS has different etiologies and a high mortality in adults.

(Rev Med Chile 2013; 141: 1475-1479)

Key words: Lymphohistiocytosis, hemophagocytic; Lymphoma; Pancytopenia.
'Sección Hematología, Hospital del Salvador, Santiago de Chile. ${ }^{2}$ Residente de Hematología, Universidad de Chile, Hospital del Salvador.

Recibido el 7 de marzo de 2013, aceptado el 9 de septiembre de 2013

Correspondencia a: Dra. Camila Peña Ojeda E-mail: camipena@gmail. com
$\mathrm{E}$ síndrome hemofagocítico (SHF) secundario, también conocido como linfohistiocitosis hemofagocítica ${ }^{1}$ es una entidad infrecuente, fatal sin tratamiento. Se debe a una descontrolada hipercitoquinemia debida a la proliferación de linfocitos y macrófagos activados. Se asocia con enfermedades subyacentes, siendo las principales algunas infecciones, enfermedades autoinmunes o neoplasias hematológicas ${ }^{2}$.

La clínica es inespecífica, destacando la fiebre, hepatoesplenomegalia, citopenias, hipertrigliceridemia, ferritina elevada, hemofagocitosis y coagulopatía. Para homogeneizar el diagnóstico comenzaron a desarrollarse criterios específicos por Henter en 1991, y luego fueron actualizados en $2004^{3}$. Es imprescindible la sospecha precoz de esta patología, ya que la evolución depende de un manejo adecuado y a tiempo. El objetivo de esta publicación es presentar y describir 5 casos de SHF secundario manejados en nuestro centro.

\section{Casos clínicos}

Se identificaron 5 pacientes, tres mujeres y dos varones, entre 17 y 51 años, con una mediana de 29 años. Las causas fueron hemato-oncológicas en 3 pacientes: linfoma $\mathrm{T}$ subcutáneo paniculitis like, linfoma del manto y linfoma anaplástico de células grandes ALK (+). En los dos primeros, el diagnóstico se realizó postmortem. En otro caso, la causa fue infección por citomegalovirus (CMV) en una paciente con trasplante hepático y en otro se atribuyó a la administración de lípidos parenterales, durante la quimioterapia de inducción de una leucemia linfoblástica aguda (LLA) de estirpe B. Con respecto a la clínica y laboratorio, todos los pacientes presentaron fiebre, citopenias, hepatoesplenomegalia y hemofagocitosis en médula ósea (Figura 1). En la Tabla 1 se resumen los parámetros estudiados en los 5 pacientes. Cuatro casos requirieron ingreso a unidad de paciente crítico, 
por requerimiento de drogas vasoactivas (DVA) y ventilación mecánica invasiva (VMI). Todos recibieron tratamiento de soporte. En cuanto al tratamiento específico de la patología subyacente del SHF en los casos que ésta se diagnosticó a

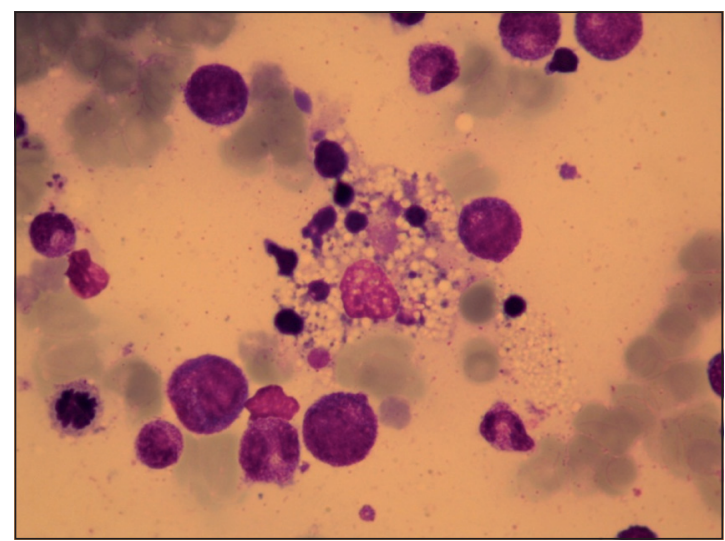

Figura 1. Hemofagocitosis en médula ósea en el paciente $n^{\circ} 5$. tiempo: el linfoma anaplástico ALK (+) se manejó con quimioterapia $\mathrm{CHOEP}$, la infección por $\mathrm{CMV}$ con ganciclovir, y se procedió a la suspensión de los lípidos parenterales, en otro caso. El manejo del SHF propiamente tal fue heterogéneo, observándose diferentes criterios en todos los pacientes. El paciente con linfoma anaplástico fue tratado con el protocolo del Hemophagocytic lymphohistiocytosis Study Group (HLH 04), simultáneamente con la quimioterapia con buena respuesta inicial, pero luego recaída del linfoma. La paciente con trasplante hepático e infección por CMV recibió ciclosporina, corticoides e inmunoglobulina endovenosa, sin respuesta. La paciente del SHF por linfoma T subcutáneo paniculitis like recibió corticoides y ciclosporina, sin respuesta. El otro paciente requirió sólo corticoides y suspensión de los lípidos parenterales, con buena respuesta. Sólo este último paciente sobrevivió. Cabe mencionar que en el paciente 4 de la Tabla 1, tanto el diagnóstico de SHF como el de linfoma se realizaron postmortem. El tiempo de sobrevida fue 75 días.

Tabla 1. Características clínicas y laboratorio de los 5 pacientes

\begin{tabular}{|c|c|c|c|c|c|}
\hline & Paciente 1 & Paciente 2 & Paciente 3 & Paciente 4 & Paciente 5 \\
\hline Sexo & M & $\mathrm{F}$ & $\mathrm{F}$ & M & $\mathrm{F}$ \\
\hline Edad & 24 & 28 & 17 & 59 & 18 \\
\hline Causa & $\begin{array}{l}\text { Lípidos en } \\
\text { parenteral (LLA) }\end{array}$ & $\begin{array}{c}\text { Linfoma T } \\
\text { paniculitis like }\end{array}$ & $\operatorname{LCGA} \operatorname{ALK}(+)$ & $\begin{array}{l}\text { Linfoma del } \\
\text { manto }\end{array}$ & $\begin{array}{l}\text { CMV en tx } \\
\text { hepático }\end{array}$ \\
\hline Fiebre & Sí & Sí & Sí & Sí & Sí \\
\hline Hepatoesplenomegalia & Sí & Sí & Sí & Sí & Sí \\
\hline $\mathrm{Hb}(\mathrm{g} / \mathrm{dL})$ & 7,6 & 9,1 & 7,8 & 6,6 & 5,2 \\
\hline RAN $\left(\mathrm{mm}^{3}\right)$ & 13 & 1.040 & 2.260 & 8.700 & 2.200 \\
\hline Plaquetas $\left(\mathrm{mm}^{3}\right)$ & 15.100 & 68.000 & 119.000 & 29.400 & 6.500 \\
\hline Triglicéridos (mg/dL) & 156 & 546 & 323 & NR & 250 \\
\hline Fibrinógeno (mg/dL) & NR & $<100$ & 221 & NR & 213 \\
\hline Hemofagocitosis MO & Sí & Sí & Sí & Sí & Sí \\
\hline Hemofagocitosis otros órganos & No & Piel & No & $\begin{array}{c}\text { Ganglio-hígado- } \\
\text { bazo }\end{array}$ & Líquido ascítico \\
\hline Ferritina (ug/L) & 825 & $>1.000$ & 1.732 & 625 & 411 \\
\hline LDH (U/L) & 378 & 740 & 777 & 4.400 & 243 \\
\hline Tpo protrombina & $61 \%$ & $40 \%$ & $63 \%$ & $42 \%$ & $11 \%$ \\
\hline Estado actual & Vivo & Fallecido & Fallecido & Fallecido & Fallecido \\
\hline
\end{tabular}

M: masculino; F femenino. LLA: Leucemia linfoblástica aguda Philadelphia (+). IgIV: gamaglobulina EV. LCGA ALK (+): linfoma células grandes anaplástico T ALK (+). tx: trasplante hepático. HLH 04: protocolo tratamiento HLH 2004. 


\section{Discusión}

El SHF secundario es una patología infrecuente y probablemente subdiagnosticada, por lo cual no existen registros de incidencia ${ }^{1}$. Una posible explicación es la semejanza del cuadro con otras situaciones clínicas, especialmente con infecciones severas. En Chile existen escasas descripciones de $\operatorname{casos}^{4}$, siendo una serie pediátrica la más numerosa $^{5}$.

Se desconoce la fisiopatología de esta enfermedad. La anormalidad principal es una hipercitoquinemia descontrolada, como resultado de una exagerada proliferación de linfocitos $\mathrm{T}$ y macrófagos activados en varios órganos ${ }^{6}$. Se ha encontrado altas concentraciones de interferon gamma, factor de necrosis tumoral, IL-6, IL 10, IL12 y del receptor soluble de IL-2 (sCD25) ${ }^{7-8}$, así como elevación de IL-18, lo que aceleraría la apoptosis de las células $\mathrm{NK}^{9}$.

Existen varias patologías subyacentes que pueden desencadenar el SHF. Las infecciones virales son las más importantes, aunque también se presentan en infecciones bacterianas, parasitarias o fúngicas. Destacan el virus Ebstein-Barr (VEB), el CMV, virus herpes simplex, virus herpes humano-8 y VIH. En nuestra serie describimos un caso de una paciente con trasplante hepático que desarrolló el SHF en relación a infección por CMV. Esta causa da cuenta de 10,5\% de SHF en inmunocomprometidos ${ }^{10}$.

Otro grupo causal importante son las neoplasias, especialmente hematológicas, como linfomas y leucemias. Esto se correlaciona con nuestra serie, ya que el linfoma constituye el diagnóstico hematológico más común. Dentro de los linfomas, el que se asocia con mayor frecuencia es el linfoma T/NK, especialmente en países orientales ${ }^{11}$. Estos linfomas tienden a responder a la terapia antineoplásica convencional, pero frecuentemente recaen ${ }^{12}$. Dentro de nuestra casuística, existen dos casos de linfomas T. El tipo paniculitis like, representa sólo $1 \%$ de los LNH, pero hasta $20 \%$ de éstos presentan SHF, lo que está asociado a peor pronóstico $^{13}$. Destaca que el SHF en síndromes linfoproliferativos B es extremadamente raro y en nuestra serie existe un caso de linfoma del manto.

En el caso de presentarse en enfermedades autoinmunes, la entidad suele llamarse síndrome de activación macrofágica. La artritis reumatoide juvenil es una causa importante, presentándose en 10-30\% de los casos. También destacan la enfermedad de Still, artritis reumatoide, lupus eritematoso sistémico o el síndrome de Sjögren ${ }^{6}$. No encontramos casos reumatológicos en nuestra serie, probablemente por subdiagnóstico.

Entre las causas misceláneas, está la asociación con medicamentos quimioterápicos o la infusión de lípidos parenterales, concordante con uno de los casos descritos, cuyo cuadro de SHF se presentó con clínica más leve.

Para el diagnóstico se usan los criterios propuestos por la "Study Group of the Histiocyte Society", que se revisaron el año 2004 y luego el $2007^{14}$. Estos se muestran en la Tabla 2. La presencia de estos criterios no han sido evaluados sistemáticamente en adultos y no siempre se cumplen todos, especialmente en los casos de autoinmunidad ${ }^{15}$. En nuestros casos todos cumplieron con 5 criterios por lo menos.

La ausencia de un cuadro febril prolongado hace muy poco probable el diagnóstico. Aproximadamente $60 \%$ de los pacientes presentan hepatoesplenomegalia. La mitad puede presentar adenopatías. También presentan eritema maculopapular, alteración de pruebas hepáticas y de coagulación. Las anormalidades neurológicas son más frecuentes en niños y se presentan en alrededor de $30 \%{ }^{6}$. Todos estos síntomas y signos se pueden explicar por el aumento de las citoquinas y la infiltración macrofágica. Es así como la fiebre se debe a la IL1 y a IL6. La hepatoesplenomega-

Tabla 2. Criterios diagnósticos para síndrome hemofagocítico. Deben estar presentes al menos 5 de ellos

\begin{tabular}{|lc|}
\hline Criterio & Valor \\
\hline Fiebre & \\
\hline Esplenomegalia & \\
\hline Citopenias $>2$ líneas & $\mathrm{Hb}<9 \mathrm{~g} / \mathrm{dL}$ \\
& $\mathrm{Plaquetas}<100.000 \mathrm{~mm}^{3}$ \\
& $\mathrm{RAN}<1.000 \mathrm{~mm}^{3}$ \\
Hipertrigliceridemia & $\geq 265 \mathrm{mg} / \mathrm{dl}$ \\
Hipofibrinogenemia & $<150 \mathrm{mg} / \mathrm{dl}$ \\
Hemofagocitosis MO, gg u otro \\
Ferritina aumentada \\
sCD25 elevado & $\geq 500 \mu \mathrm{g} / \mathrm{l}$ \\
Actividad NK & $\geq 2.400 \mathrm{U} / \mathrm{ml}$ \\
\hline
\end{tabular}

MO: médula ósea; gg: ganglio. 
lia, la alteración de las pruebas hepáticas (que se presenta en 60-90\%) y los síntomas neurológicos son la consecuencia de infiltración de órganos por linfocitos y macrófagos ${ }^{16}$.

En cuanto al laboratorio, se encuentra citopenias, generalmente trombocitopenia y anemia, consecuencia del aumento del factor de necrosis tumoral alfa (FNT- $\alpha)$. La hipertrigliceridemia se debe a la inhibición de la lipasa lipoproteica por el FNT- $\alpha$. El aumento de la ferritina y de sCD25 se debe a que forman parte de las proteínas de fase aguda y podría significar severidad de la enfermedad ${ }^{17}$. La hipofibrinogenemia se describe en la mayoría de los pacientes, debido a la liberación de activador del plasminógeno por macrófagos estimulados ${ }^{5}$.Todos nuestros casos presentaron fiebre, esplenomegalia, citopenias y hemofagocitosis. Cabe destacar que esta última no es necesaria para el diagnóstico, dado que al comienzo de la enfermedad puede ser inexistente. Destaca además que en algunos casos se demostró hemofagocitosis en otros órganos, como hígado, bazo, ganglios y líquido ascítico (Tabla 1).

Tal como se describe en la literatura, los casos descritos se presentaron como cuadros clínicos graves, con falla multiorgánica, por lo que cuatro requirieron ingreso a unidad de cuidados intensivos.

La mortalidad es elevada, entre 20 y $80 \%$, dependiendo de la causa desencadenante: 20$40 \%$, cuando se asocia a infección y de casi $100 \%$, cuando se asocia a patologías malignas. En nuestra serie, la mortalidad de $80 \%$, puede explicarse por la alta incidencia de causas neoplásicas.

El tratamiento se basa en suprimir la activación exagerada del sistema inmune. En los casos de SHF familiar se basa en el protocolo HLH $04^{3}$, un protocolo pediátrico, que se usa como puente para trasplante alogénico. En el sistema público chileno, esta alternativa terapéutica parece poco factible en adultos, ya que el síndrome hemofagocítico no constituye una indicación para ello. El tratamiento del SHF secundario en adultos no está claro. Se basa en discusión caso a caso. Se utiliza dexametasona basado en su habilidad se cruzar la barrera hematoencefálica y su indicación principal es en linfomas y enfermedades autoinmunes. El uso de etopósido ha sido efectivo en casos de SHF asociado a $\mathrm{VEB}^{18}$. La ciclosporina ha sido efectiva en casos de infecciones virales y autoinmunes. La inmunoglobulina (Ig) iv se ha usado en SHF por
CMV con buenos resultados ${ }^{19-20}$. Actualmente, se investiga terapias target como anticuerpos anticitoquinas y terapia génica ${ }^{6}$.

Lamentablemente el tratamiento de los 5 pacientes fue diferente, por lo que no podemos sacar conclusiones. El único paciente que sobrevive, fue el que se asoció a la administración de lípidos parenterales y que respondió rápidamente a corticoides y la suspensión de los lípidos.

Se requieren estudios con mayor número de pacientes para evaluar el tratamiento óptimo.

Creemos importante la publicación de estos casos, ya que una sospecha precoz junto al inicio del tratamiento respectivo podrían contribuir a un pronóstico favorable, lo cual depende fundamentalmente de la etiología subyacente.

\section{Referencias}

1. Emmeneggera U, Schaerb D, Larrochec C, Nefteld K. Haemophagocytic syndromes in adults: current concepts and challenges ahead. Swiss Med Wkly 2005; 135: 299-314.

2. Larroche C. Hemophagocytic lymphohistiocytosis in adults: Diagnosis and treatment. Joint Bone Spine 2012; 79: 356-61.

3. Henter JI, Tondini C, Pritchard J. Histiocyte disorders. Crit Rev Oncol Hematol 2004; 50: 157-74.

4. González B, Roa, Schmidt N. Síndrome de activación macrofágico en pediatría: A propósito de cuatro casos. Rev Chil Pediatr 2005; 76 (2); 183-92.

5. Verdugo P, Rodríguez N, Tordecilla J, Soto V. Síndrome hemofagocítico secundario en pediatría. Experiencia clínica en ocho casos. Rev Chil Pediatr 2005; 76: 397-403.

6. Bode S, Lehmberg K, Maul-Pavicic A, Vraetz T, Janka $\mathrm{G}$, Stadt $\mathrm{U}$, et al. Recent advances in the diagnosis and treatment of hemophagocytic lymphohistiocytosis. Arthritis Res Ther 2012; 14: 213.

7. Takada H, Ohga S, Mizuno Y. Increased IL-16 levels in hemophagocytic lymphohistiocytosis. J Pediatr Hematol Oncol 2004; 26: 567-73.

8. Grom AA. Macrophage activation syndrome and reactive hemophagocytic lymphohistiocytosis: the same entities? Curr Opin Rheumatol 2003; 15: 587-90.

9. Mazodier K, Marín V Novick D, Farnarier C, Robitail S, Schleinitz N, et al. Severe imbalance of IL-18/IL-18BP in patients with secondary hemophagocytic syndrome. Blood 2005; 106: 3483-9.

10. Fisman DN. Hemophagocytic syndromes and infection. Emerg Infect Dis 2000; 6: 601-8.

11. Tong H, Ren Y, Liu H, Xiao F, Mai W, Meng H, et al. 
Clinical characteristics of T-cell lymphoma associated with hemophagocytic syndrome: Comparison of T-cell lymphoma with and without hemophagocytic syndrome. Leuk Lymphoma 2008; 49: 81-7.

12. Han AR, Lee HR, Park BB, Hwang IG, Park S, Lee S, et al. Lymphoma-associated hemophagocytic syndrome: clinical features and treatment outcome. Ann Hematol 2007; 86: 493-8.

13. Koh MJ, Sadarangani SP, Chan YC, Chan MY, Tan AM, Tan SH, et al. Aggressive subcutaneous panniculitislike T-cell lymphoma with hemophagocytosis in two children (subcutaneous panniculitis-like T-cell lymphoma). J Am Acad Dermatol 2009; 61: 875-81.

14. Henter JI, Horne A, Arico M. Diagnostic and therapeutic guidelines for hemophagocytic lymphohistiocytosis. Pediatr. Blood Cancer 2007; 48: 124-31.

15. Parodi A, Dav`1 S, Pringe AB, Ruperto N, MagniManzoni S, Miettunen P, et al. Macrophage activation syndrome in juvenile systemic lupus erythematosus: a multinational multicenter study of thirty-eight patients. Arthritis Rheum 2009; 60: 3388-99.

16. Janka G. Hemophagocytic syndromes. Blood Reviews 2007; 21: 245-53.

17. Filipovich A. Hemophagocytic Lymphohistiocytosis and Other Hemophagocytic Disorders. Immunol Allergy Clin N Am 2008; 28: 293-313.

18. Imashuku S. Treatment of Epstein-Barr virus-related hemophagocytic lymphohistiocytosis (EBV-HLH); update 2010. J Pediatr Hematol Oncol 2011; 33: 35-9.

19. Hot A, Madoux MH, Viard JP, Coppéré B, Ninet J. Successful treatment of cytomegalovirus-associated hemophagocytic syndrome by intravenous immunoglobulins. Am. J. Hematol 2008; 83: 159-62.

20. Gill DS, Spencer A, Cobcroft RG. High-dose gammaglobulin therapy in the reactive haemophagocytic syndrome. Br J Haematol 1994; 88: 204-6. 